\title{
Analysis of Patterns of Adverse Transfusion Reactions and Management: A Novel Initiative toward Hemovigilance in a Teaching Hospital of South India
}

\author{
Anandraj Vaithy K. ${ }^{1} \quad$ Janani Mathialagan ${ }^{1} \quad$ A.T.M. Venkat Raghavan ${ }^{1}$ Sowmya Srinivasan ${ }^{1}$ \\ ${ }^{1}$ Department of Pathology, Mahatma Gandhi Medical College \& \\ Research Institute, Sri Balaji Vidyapeeth University (Deemed), \\ Puducherry, India

\begin{abstract}
Address for Correspondence Anandraj Vaithy K., MD Department of Pathology, Mahatma Gandhi Medical College \& Research Institute, Sri Balaji Vidyapeeth University (Deemed), Puducherry 607402,
\end{abstract} \\ India (e-mail: kanandrv@gmail.com).
}

\begin{abstract}
Objective Blood transfusion being a major lifesaving frontline procedure in majority of clinical wards, has a high potential with risk for adverse events ranging in severity, thus warranting optimal usage of blood. Continuous monitoring of adverse transfusion reaction (ATR) and analysis of the reactions can promote better comprehension of various contributing risk factors. Hemovigilance being a quality indicator with data system assists in ensuring quality and safe blood transfusion. The present study was designed to analyze the incidence and nature of adverse transfusion-associated events reported to the blood bank from our teaching hospital located in the remote part of South-East Coastal region with emphasis on corrective and preventive actions.

Materials and Methods The prospective study included all the ATR reported to our hospital blood bank over a period of 42 months. The transfusion reactions report forms filled by the clinicians were analyzed and the reactions were categorized into patterns. The associated risk factors for the reaction are evaluated with substantial laboratory and clinical parameters in reference to hemovigilance.

Results Among 19,800 transfusions, 189 transfusion reactions were encountered with a ratio of 10:0.9 with age ranging from 12 to 80 , with female preponderance. Reactions associated with whole blood (WB) transfusion were common ( $50 \%, p=0.002)$ followed by packed red blood cells transfusion (PRBC) $(1.4 \%, p=0.006)$ and platelets $(0.8 \%, p=0.1)$. Febrile nonhemolytic transfusion reactions were predominant patterns noted in 122 cases followed by allergic reactions in 43 cases with more commonly associated PRBC transfusion whereas hemolytic reactions showed association with WB transfusion. No delayed reactions or mortalities were encountered.

Conclusion The present study concludes with novelty showing the overall incidence

Keywords

- adverse transfusion reaction

- hemovigilance

- hemolytic reactions

- quality assurance of transfusion-associated reactions in the study domain is slightly higher than more advanced centers in India owing to lack of leukodepletion units which could enable to substantially retard the incidence of adverse events of transfusion as a general measure and febrile reactions in particular. Hemovigilance and pattern analysis of ATR provide scope for corrective and preventive action and give referral guidelines to prevent future recurrence.
\end{abstract}

DOI https://doi.org/

$10.1055 / \mathrm{s}-0040-1716592$

ISSN 0974-2727. (c)2020 by The Indian Association of Laboratory Physicians
License terms

(ㅇ)(1) $\Theta \circledast$ 


\section{Introduction}

Blood transfusion remains as a mainstay of treatment among clinical disciplines in the process of managing elective and crucial cases. ${ }^{1}$ Appropriate and adequate access to safe uneventful blood transfusion resources is considered as an integral part to any basic health care delivery system. ${ }^{1}$ Researchers quote an efficient blood banking system serves as a mirror of the hospital quality system. ${ }^{1,2}$ Transfusion medicine often takes a frontline lead serving as a lifesaving process especially among critically ill patients. ${ }^{3}$ However, transfusion of blood and its components is inherent with its own risks ranging from minor complications to life threatening fatal conditions which are broadly coined as "adverse transfusion reactions (ATRs)." Rationale selection of patient with pragmatic assessment of pretransfusion parameters aids to reduce the incidence rate of adverse reactions. ${ }^{1,4}$ It also provides scope to analyze risk versus beneficiary outcome of transfusion thereby picking up potential recipient to contain transfusion-related adverse reactions. ${ }^{5}$ This continuous monitoring of ATR serves as an effective modality in promoting quality control in terms of safety and patients care.

Adequate knowledge with regard to ATRs and its various patterns of presentation will guide for prompt identification with subsequent management and appropriate measures to prevent ATR in future as well. ${ }^{1,6}$ Hemovigilance being a quality indicator of transfusion medicine, functions as a continuous system of data collection for analysis of transfusion-related adverse reactions. ${ }^{6,7}$ It provides a platform to investigate their underlying etiopathogenesis and clinical outcomes and prevent their occurrence as well as recurrence in long term. ${ }^{7,8}$ Thus hemovigilance process is directed with a purpose to ensure safety and efficacy of blood transfusion procedure covering the sequential chain of donors to recipients. ${ }^{9,10}$

Hemovigilance based on its core principle includes proper identification, reporting, reverting, repeating investigations, and tracking various chain of events between donors and recipients. ${ }^{11,12}$ It also interrogates incidents occurred in manufacturing level and consequent errors and "near misses."13,14 In the year 2012, "the Haemovigilance Programme of India" was commissioned by the Indian Pharmacopoeia Commission in collaboration with the National Institute of Biologicals under the auspices of Pharmacovigilance Programme of India, with an objective to track ATR due to transfusion of blood and its components in all affiliated blood banks across the country. ${ }^{6,15}$

Hemovigilance system serving as a backbone of quality assurance, the present study was carried out with a novel aim of determining incidence of adverse transfusion-related reactions and to substantiate its patterns encountered in our teaching hospital blood bank located in remote SouthEastern part of the Country. The study owns an objective to enlighten knowledge and insights into patterns of ATR in the study domain which is not yet documented with special emphasis on root cause analysis with appropriate corrective and preventive actions.

\section{Materials and Methodology}

\section{Study Design and Duration}

The observational study was conducted prospectively in our Blood Bank, Department of Pathology, in teaching hospital located in remote part of South-East Coastal region for a duration of 3 years and 6 months from March 2016 to October 2019. After approval from Institute of Research Board Ethical Committee, the number of units of blood and its components issued, incidence of adverse events of transfusions, and type of reactions are documented in a format. Hospital Laboratory Information system-AOSTA backbone software was used to retrieve all laboratory data available both pre and post transfusion for the study cases.

\section{Standard Operating Procedure for Adverse Event Reporting, Prevention, Detection, and Evaluation}

The hospital blood bank strictly adheres to the standard operating procedure guidelines proposed by authority bodies like Central Drugs Standard Control Organization and National AIDS Control Organization (NACO) manual for performing transfusion including donor selection, phlebotomy procedures, component processing and separation, laboratory testing for infectious diseases, storage of units, cross matching, issue of component, and post-transfusion follow-up of the recipient. All the concerned health care workers involved in transfusion procedure including clinicians, paramedical staff have been trained in transfusion protocols thereby making them aware to identify, report, and manage any adverse events encountered at the earliest possible.

Pretransfusion training included logistical work-up like cross checking for any possible clerical errors, blood group of the recipient (ABO-Rh system), type of transfusion (whole blood or components), visual inspection of blood bag for any color change or evidence of hemolysis, blood clots and spillage, date of bleeding, and expiry date. Tube method of blood grouping being the gold standard method was routinely followed in our blood bank. The training also sensitized the concerned staff on safe transfusion protocol and time duration for transfusion such as whole blood and packed red blood cells (PRBCs) must be started within 30 minutes of release and to be completed before 4 hours while platelets concentrates and fresh frozen plasma must be transfused instantly and completed within 20 minutes duration.

\section{Reporting of Events of ATR}

All the blood bag units issued were attached with transfusion reaction reporting form (TRRF) and in case of any adverse events, clinicians were instructed to duly fill up the form and return it back to blood bank along with left remnant blood product from the used bag and blood sets. After immediate reporting of events, the ward staffs were instructed to collect two blood samples (one in ethylenediaminetetraacetic acid vial and one in plain vial, each $2 \mathrm{~mL}$ ) drawn from the limb opposite to transfusion and first urine sample voided after post transfusion. Our study adapted the concept of 
3R-"Report, Revert, and Repeat" in a sequential flow as per standard protocol of error management.

Report: All the reported reactions were evaluated as per transfusion reaction work-up form proposed by National Institute of Biologicals (NIB), Ministry of Health and Family Welfare, Government of India. ${ }^{1}$

Repeat: On receiving the event report, the blood bank faculty performed extensive complete evaluation including rechecking the patient's identity both pre and post transfusion vials, possibility for mismatch sampling, erroneous transposition, blood group (ABO-Rh), and screening for antibodies. Blood grouping and typing were again repeated and compared with the pretransfusion records and documented. Clinical symptoms like fever, chills and rigors, hypotension sequelae, rigors, skin rashes/petechiae, dyspnea, or any relevant untoward events noted during the course and following transfusion were documented in the classification of transfusion reaction patterns.

\section{Laboratory Investigations}

The blood bag was inspected for any gross evidence of hemolysis, clots, and discoloration. In such case of suspected hemolytic reaction, further confirmatory laboratory investigations were done like qualitative and quantitative plasma hemoglobin estimation, direct antiglobulin test, urine examination for hemoglobinuria and hematuria, liver function test or LFT (serum total and unconjugated bilirubin), renal function test, and peripheral blood smear examination (to look for presence of schistocytes/spherocytes). Compatibility testing was repeated once again on both pre and post transfusion samples

Microbiological examination of the bag was performed from the patient's blood for culture studies to rule the possibility of any bacteriological infections.

\section{Statistical Analysis}

The data and parameters collected for the study were evaluated in terms of frequency (\%), mean, and standard deviation. The parameters were entered in Microsoft Office Excel sheet 2010 and SPSS (Statistical Package for Social Sciences) was used for statistical analysis. Chi-square test was applied and $p$-value $<0.05$ was taken to be significant.

\section{Results and Observation}

The present prospective study was conducted for a period of 3 years and 6 months and ATRs reported to the blood bank were documented and analyzed on the grounds of their clinical features and laboratory investigations. A total of 19,800 units of blood and components derived from 6,424 units collected from donors were issued for transfusion to patients admitted under various clinical specialties. Among the 19,800 units transfused, ATRs were encountered in 189 patients (0.95\%) with transfusion rate to ATR ratio of 10:0.1. Based on the clinical signs and symptoms mentioned in TRRF, transfusion medicine evaluation interpretation and results of various laboratory investigations, the ATRs were categorized into standard criteria as defined by the Hemovigilance Centre, NIB, ${ }^{1}$ as shown in - Table 1 .

The age of the patients who underwent transfusion ranged between 12 and 81 years with female preponderance of 117 cases (61\%) compared with 70 male cases (38\%) and two transgender cases (1\%). However, the gender difference does not seem to show statistical significance ( $p$-value $\left.=0.09, x^{2}=2.36\right)$. Similarly, high incidence of ATR occurred in recipients with AB positive group of 65 cases (34\%). The demographic characteristics of ATR are tabulated in - Table 2.

PRBC transfusion showed common association with adverse reactions in 126 cases (66\%) and whole blood nine cases (50\%) followed by platelet concentrates (PC) 36 cases

Table 1 Categorization of adverse transfusion reaction

\begin{tabular}{|l|l|l|}
\hline $\begin{array}{l}\text { Type of } \\
\text { reaction }\end{array}$ & $\begin{array}{l}\text { Acute (onset } \\
\text { within }<24 \mathrm{~h} \\
\text { duration after } \\
\text { cessation of } \\
\text { transfusion) }\end{array}$ & $\begin{array}{l}\text { Delayed (onset } \\
\text { after }>24 \mathrm{~h} / \mathrm{d} \\
\text { after cessation of } \\
\text { transfusion) }\end{array}$ \\
\hline $\begin{array}{l}\text { Immune } \\
\text { mediated }\end{array}$ & $\begin{array}{l}\text { Hemolytic febrile, } \\
\text { nonhemolytic aller- } \\
\text { gic/anaphylactic } \\
\text { transfusion-related } \\
\text { acute lung injury } \\
\text { (TRALI) }\end{array}$ & $\begin{array}{l}\text { Hemolytic reaction } \\
\text { alloimmunization, RBC } \\
\text { antigens graft vs. host } \\
\text { disease post transfusion } \\
\text { purpura. }\end{array}$ \\
\hline $\begin{array}{l}\text { Nonimmune } \\
\text { mediated }\end{array}$ & $\begin{array}{l}\text { Transfusion- } \\
\text { associated circu- } \\
\text { latory overload } \\
\text { embolism (air) } \\
\text { hyperkalemia } \\
\text { hypotension. }\end{array}$ & $\begin{array}{l}\text { Iron overload } \\
\text { Malaria and infections }\end{array}$ \\
\hline
\end{tabular}

Abbreviation: RBC, red blood cell.

Table 2 Distribution of demographic characteristics of ATR $(n=189)$

\begin{tabular}{|c|l|}
\hline Parameters & $\begin{array}{l}\text { Frequency of } \\
\text { ATR }\end{array}$ \\
\hline Gender & \\
\hline Males & $70(61)$ \\
\hline Females & $117(38)$ \\
\hline Transgender & $02(1)$ \\
\hline Age (in y) & \\
\hline Range & 1281 \\
\hline Mean age & $52 \pm 22.5$ \\
\hline Blood group (Tube method) & \\
\hline AB positive & $65(34 \%)$ \\
\hline A positive & $35(18.5)$ \\
\hline B positive & $50(26.5)$ \\
\hline O positive & $39(21)$ \\
\hline
\end{tabular}

Abbreviation: ATR, adverse transfusion reaction.

Note: Figures in parenthesis indicate frequency in terms of percentage $(\%)$. 
(19\%) and others as shown in - Table 3. No adverse reactions were noted with cryoprecipitate and cryopoor plasma transfusion. On auditing the requests, maximum requests were from Obstetrics and Gynecology Department, i.e., 8,104 requests (40\%) showing higher incidence of ATR 117 cases (61.9\%) followed by other departments as shown in - Table 4.

Time interval of transfusion: Among 19,800 transfusions, 189 patients had ATR (0.9\%). In case of PRBC (8,910 units) and whole blood transfusion (18 units), the time interval between the issue of blood issue and transfusion of the implicated unit ranged between 30 minutes and 6 hours duration. It had been observed that during this transition period, the units were in state of unmonitored conditions in clinical wards and theatres. Platelet units $(n=4,410)$, were transfused in with a time range of 25 to 45 minutes where the platelet units were found to be placed at patient's bedside in substantial cases.

\section{Categorization of ATR Patterns}

The adverse reactions were classified into acute (within 24 hours) and delayed (onset after a period of 24 hours from cessation of transfusion). With regard to clinical presentation, febrile nonhemolytic transfusion reactions (FNHTR) constituted majority of the ATR commonly associated with PRBC transfusion followed by allergic reactions and hypotension. The distribution of adverse reaction patterns observed with respect to transfusion of blood and its components is depicted in - Table 5

All the cases in the study ( $n=189$ cases) were acute transfusion reactions, where 122 (69\%) cases showed FNHTR, 43 cases (21\%) showed allergic reactions, 18 cases (8\%) showed hypotension, and six (2\%) cases showed AHTR. Majority of transfusion reaction occurred in patients with anemia as indicated (90 cases) followed by dialysis ( 59 cases), elective surgical procedures (27 cases), and others (13 cases) as shown in - Fig. 1.

Table 3 Frequency of blood units issued vs. ATR encountered during study period

\begin{tabular}{|l|l|l|l|}
\hline Blood derivative & $\begin{array}{l}\text { Number of blood units } \\
\text { transfused }\end{array}$ & ATR & $\begin{array}{l}\text { Statistical analysis } \\
p \text {-Value }\end{array}$ \\
\hline PRBC & $8,910(45)$ & $126(1.4)$ & $p=0.06,\left(x^{2}=2.91\right)$ \\
\hline FFP & $6,300(31.8)$ & $18(0.2)$ & $p=0.09,\left(x^{2}=0.81\right)$ \\
\hline PC & $4,410(22.2)$ & $36(0.8)$ & $p=0.10,\left(x^{2}=0.21\right)$ \\
\hline WB & $18(0.09)$ & $09(50)$ & $p=0.002,\left(x^{2}=0.04\right)$ \\
\hline Cryoprecipitate & $153(0.7)$ & NR & NA \\
\hline CPP & $09(0.045)$ & NR & NA \\
\hline Total & 19,800 & 189 & - \\
\hline
\end{tabular}

Abbreviations: ATR, adverse transfusion reaction; CPP, cryopoor plasma; FFP, fresh frozen plasma; NA, not applicable; NR, not reported; PC, platelet concentrates; PRBC, packed red blood cells transfusion.

Note: Figures in parenthesis indicate frequency in terms of percentage (\%) with overall all units transfused.

Table 4 Categorization of ATR reported in respect to audit of requests for clinical departments

\begin{tabular}{|c|c|c|c|c|}
\hline Clinical departments & Type of component & $\begin{array}{l}\text { Number of blood } \\
\text { units issued }\end{array}$ & ATR & Total ATR \\
\hline \multirow[t]{5}{*}{ Obstetrics and Gynecology } & PRBC & 7200 & 99 & \\
\hline & PC & 900 & 14 & 117 \\
\hline & WB & 04 & 04 & \\
\hline & PRBC & 900 & 11 & 27 \\
\hline & FFP & 1800 & 02 & \\
\hline \multirow[t]{3}{*}{ General surgery } & PC & 1530 & 09 & \\
\hline & WB & 12 & 05 & \\
\hline & PRBC & 810 & 16 & \\
\hline \multirow[t]{3}{*}{ General medicine } & FFP & 4500 & 16 & 45 \\
\hline & PC & 1980 & 13 & \\
\hline & WB & 02 & 00 & \\
\hline Total & & 19800 & 189 & 189 \\
\hline
\end{tabular}

Abbreviations: FFP, fresh frozen plasma; PC, platelet concentrates; PRBC, packed red blood cells transfusion; WB, whole blood. 
Table 5 Incidence of patterns of ATR in respect to transfusion of blood products

\begin{tabular}{|c|c|c|c|c|c|c|}
\hline \multirow{2}{*}{$\begin{array}{l}\begin{array}{l}\text { Number of } \\
\text { transfusion }\end{array} \\
\text { Blood } \\
\text { derivatives }\end{array}$} & \multirow[b]{2}{*}{ FNHTR } & \multicolumn{5}{|c|}{ Patterns of ATR $(n=189)$} \\
\hline & & $\begin{array}{l}\text { Allergic } \\
\text { reactions }\end{array}$ & Hypotension & AHTR & $\begin{array}{l}\text { Total ATR with } \\
\text { blood } \\
\text { /components }\end{array}$ & Risk ratio \\
\hline WB $(n=18)$ & 3 & 2 & - & 4 & 09 & 0.50 \\
\hline PRBC $(8,910)$ & 90 & 20 & 9 & 2 & 126 & 0.02 \\
\hline $\mathrm{PC}(n=4,410)$ & 18 & 14 & 9 & - & 36 & 0.009 \\
\hline $\operatorname{FFP}(6,300)$ & 11 & 7 & - & & 18 & 0.004 \\
\hline Total & 122 & 43 & 18 & 06 & 189 & \\
\hline
\end{tabular}

Abbreviations: AHTR, acute hypotensive transfusion reaction; ATR, adverse transfusion reaction; FFP, fresh frozen plasma; FNHTR, febrile nonhemolytic transfusion reactions; PC, platelet concentrates; PRBC, packed red blood cells; WB, whole blood.

\section{Transfusion rate with respect to clinical indications}

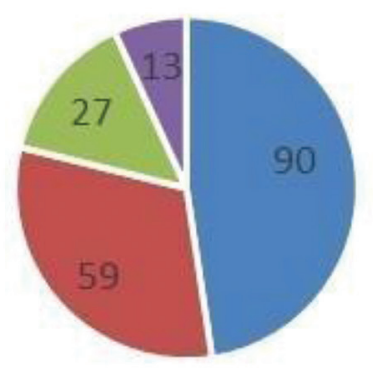

\section{- Anemia Dialysis Elective surgeries Emergency trauma care}

Fig. 1 Distribution of frequency clinical indications for blood transfusion.

\section{Febrile Nonhemolytic Transfusion Reaction}

A total of 122 cases had clinical features of febrile illness without hemolysis noticed in males (90 cases), females (30 cases), and transgender (two cases). Age incidence ranged between 45 and 70 years and fever was the most common clinical symptom followed by chills and rigors.

Allergic reactions were observed in 43 cases ( 25 females and 18 males) especially among middle age (30-45 years) and were encountered in form of urticaria (18 cases), itching (ten cases), and rashes (15 cases).

Acute hemolytic transfusion reaction: Among six cases of hemolytic reaction, evidence of hemolytic changes was noted in whole blood (four cases) and packed cells (two cases). Whole blood transfusion had high risk for adverse reactions with potential to cause febrile hemolytic transfusion reaction (FHTR) as noticed in six cases $(n=18)$ in form of hemolysis which was confirmed by appropriate laboratory tests by the presence of peripheral smear schistocytes (three cases), RBC cast in urine sediment (two cases), and direct Coombs test (four cases). Patients with FHTR showed abnormal LFT in the form of elevated S. bilirubin (three cases) and increased alkaline phosphatase levels. The laboratory investigations results and work-up for ATR were shown in - Table 6.

\section{Discussion}

Quality assurance being an integral part of efficient blood banking system, prompt reporting by clinicians on information related to any adverse events encountered during or post transfusion is the main source to document ATRs. ${ }^{1-5}$ In the present study, the adverse reactions were of acute nature with a transfusion to ATR ratio of 10:0.1 (0.9\%), concording with prior studies performed in Northern parts of Chandigarh and Punjab where the incidence of ATR was noted to be $1.09 \%{ }^{6,7}$ Observations from two long-term studies conducted in the domain of New Delhi and Chandigarh documented much lower frequency of ATR (0.05 and $0.19 \%$, 
Table 6 Comparison of results of laboratory investigations from post ATR samples

\begin{tabular}{|l|l|l|l|}
\hline \multirow{2}{*}{ Lab investigations } & \multicolumn{2}{|c|}{ FHTR (No. of cases) ( $\boldsymbol{n}=\mathbf{0 6})$} & FNHTR (No. of cases) ( $\boldsymbol{n}=\mathbf{1 8 3})$ \\
\cline { 2 - 4 } & Peripheral smear for schistocytes & $\mathbf{0 3}$ & - \\
\hline Hematology & Increased plasma Hemoglobin & 02 & - \\
\hline & Discoloration of blood & 06 & 10 \\
\hline Urine examination & Blood mixed appearance & 04 & 02 \\
\hline (Post & $\begin{array}{l}\text { Microscopy } \\
\text { RBC cast }\end{array}$ & 02 & - \\
\hline transfusion) & WBC cast & 01 & \\
\hline & Pus cells & 05 & 30 \\
\hline Repeat cross & RBCs & 04 & 02 \\
\hline Matching & Compatible & 04 & 171 \\
\hline Direct & Noncompatible & 02 & 11 \\
\hline Coombs test & Positive & 04 & - \\
\hline Biochemical tests & Negative & 02 & 183 \\
\hline & Abnormal liver function test & 05 & 12 \\
\hline
\end{tabular}

Abbreviations: ATR, adverse transfusion reaction; FNHTR, febrile nonhemolytic transfusion reactions; RBC, red blood cells; WBC, white blood cell.

respectively). ${ }^{7}$ Similar studies conducted in European Nations reported lower reaction rates ranging between 0.035 and $0.02 \%{ }^{8}$ Thus it is evident that adverse transfusion events at our study place was modestly higher when compared with studies performed at national and international centers. ${ }^{7-9}$ The reason for higher incidence could be altered immune system, medication history, circumstantial evidences for oncotic imbalance, thermal conditions, osmotic injuries, storage conditions of the blood unit issued, mode of transfusion, increased number of reporting and reverting, and high frequency of transfusion. ${ }^{7}$

Surprisingly, the frequency of adverse events was commoner among females compared with males with an approximate ratio of 1.5:1. Studies carried out in Indian domain predominantly showed male preponderance for ATR with an average percentage ranging between 54 and 59\%, whereas studies done in Middle-East region showed female preponderance almost concurring with ratio observed in the present study. ${ }^{7.8}$ It had been estimated that the frequency of ATR varies between $0.2 \%$ and a maximum of $10 \%$ with a mortality rate of two in $2,00,000 .^{9,10}$ The present study correlated and varied with prior studies on ATR performed among Indian population conducted in other parts of the country including premier institutions like All India Institute of Medical Sciences, is elaborated in - Table 7.

The concept of biosafety and hemovigilance had been evolved in later part of 19th century and gained popularity since its time of inception. ${ }^{1,11}$ The term "hemovigilance" is now recognized as a quality indicator and practiced worldwide as an essential component of quality assurance in transfusion medicine. ${ }^{12,13}$ It functions as a system on an ideal objective directed to detect, document, and evaluate undesirable events encountered with transfusion of blood and its components, thereby enabling to perform root cause analysis and setting up or reframing policies and improvisation of standard operating procedures. ${ }^{13,14}$

Data collected from different studies on the incidence of FNHTR, correlate and vary in equal proportion in the literatures. ${ }^{6,7}$ FNHTR is defined as a reaction otherwise unexplained with a rise in body temperature of minimum $1^{\circ} \mathrm{C}$ while the transfusion is ongoing or shortly after cessation of transfusion. ${ }^{1,15}$ The potential reason for this variations is attributed to several possible factors such as monitoring clinical signs and symptoms by the bedside clinical staff nurses and residents, alteration of internal temperature due to pretransfusion of medicines, case ascertainment, environment, geographical distributions, and racial inheritance.,16 In the present study, the incidence of FNHTR was high (64\%) especially in cases with PRBC transfusion. The association between FNHTR with preceding PRBC transfusion showed significant statistical analysis ( $p$-value $<0.05$ ) concurring with prior studies as well.,17 The reason attributed for high adverse events with PRBC association is higher frequency of transfusion compared with other components and majority of cases had prior transfusion leading to antigenic exposure. ${ }^{18}$ PRBC being non-leukodepleted component, has higher potential for ATR as evident from our study. To manage the high incidence of FNHTR, the study proposes to first rule possibility of hemolysis, to inject antihistamines and in case if reaction occurs for second time, usage of leukodepleted blood components must be encouraged. ${ }^{1,18}$

In reference to audit of requests from clinical departments, Obstetrics and Gynecology topped the table in claiming for maximum requests with an overall issue rate of 8,100 among which issue for PRBC with anemia as a common underlying indication. ${ }^{15}$ Most of the cases had multiple transfusions leading to adverse reaction due to varied antigenic exposure and same is the factor for adverse reactions among females. 
Table 7 Comparison of ATR observed in reference to prior Literatures

\begin{tabular}{|l|l|l|l|l|}
\hline Author of the study & Whole blood & PRBC & Platelets & FFP \\
\hline Kumar et al $^{7}$ & $45 \%$ & $12 \%$ & $18.4 \%$ & $19.3 \%$ \\
\hline Venkatachalapathy $^{9}$ & $21 \%$ & $25 \%$ & - & $2.08 \%$ \\
\hline Haslina et al $^{10}$ & $30 \%$ & $22 \%$ & $72 \%$ & $16.9 \%$ \\
\hline Bhattacharya et al $^{11}$ & $52 \%$ & $19 \%$ & $6.57 \%$ & $7.4 \%$ \\
\hline Present study & $50 \%$ & $14 \%$ & $2.52 \%$ & $9 \%$ \\
\hline
\end{tabular}

Abbreviations: ATR, adverse transfusion reaction; FFP, fresh frozen plasma; PRBC, packed red blood cells

Allergic reaction was the second highest prevalence of adverse reaction observed to be associated with transfusion and it is noted in 43 cases ( $0.2 \%$ of overall transfusion) presenting as urticaria and rashes. Statistical analysis revealed insignificant difference between the frequency of allergic events and type of blood transfused ( $p$-value $>0.05$ for all blood components). Few studies had been conducted by Kumar et al in Northern part of India at AIIMS, New Delhi, which showed that overall incidence of allergic reaction is only $0.02 \%$ which is significantly lower than our observed value. ${ }^{7}$ Studies had showed that allergic reactions can occur in from 0.1 to $2 \%$ of transfusion at a given point of time concurring with the observations of the present study. ${ }^{7}$ The pathological sequence associated with allergic reactions is due to donor's plasma interacting antigen/allergens in patient plasma and with subsequent triggering of histamine action and release of chemical mediators of inflammation. Another significant contributing factor for high allergic reaction assumed to the remnant plasma kept in PRBC to decrease the blood viscosity may expedite immune reactions. ${ }^{8,18}$ The high incidence could be fixed by administering antihistamines at least 1 hour prior to transfusion..$^{18}$ Platelet transfusions showed reactions with an incidence of $0.8 \%$ in overall transfusion which is still higher when compared with other Indian studies which showed an incidence of $0.04 \% .711$

The practice of whole transfusion should be restricted until it is absolutely indicated and NACO proposes that in a given year the utilization or issue of whole blood must be less than 10 percentage of overall issues of the blood bank. ${ }^{1}$ In the present study hemolytic reaction was commonly associated with whole blood transfusion in four cases $(22 \%, n=18)$ and two cases with PRBC (0.02\%). Laboratory investigations showed the presence of schistocytes on blood smear indicating evidence of hemolysis and with additional evidences for urine RBC cast and positive direct Coombs test. In the study, we re-emphasized the clinician regarding the management protocol in event of hemolytic reactions to inform the blood bank promptly to disconnect the entire infusion, to collect a post transfusion vial fresh blood, and to return the blood bag immediately to the blood bank.

Hypotension was encountered in 18 cases with PRBC and platelet concentration each with distribution of nine cases and the patients were already on treatment with ACE inhibitors and on dialysis. PC transfusion showed adverse events in 36 cases (0.8\%) with FNHTR and allergic reactions and the study proposed administration of corticosteroids and also to monitor the platelet counts since, many a times thrombocytopenia is a self-limiting condition and platelet transfusion is nonbeneficial. ${ }^{19}$

Though not encountered in the study, as an additional novelty, the study proposes management protocol in instances of delayed transfusion reactions as it is neither feasible to follow-up patients once they get discharged nor prevent them from succumbing to delayed reactions. We propose that whenever RBC antibodies are detected in blood bank, the treating physician should be kept informed about the same and to counsel them to provide this vital information to the patients when they get hospitalized elsewhere to avoid delayed reactions. In the present study, delayed transfusion reactions were not encountered probably due to nonreporting, due to loss of follow-up, under diagnosis since the recipients get discharged with a time interval to visit for reviews.

Critical appraisal: The study claims novelty by advocating the practice of nonpunitive type of error management in managing events of adverse transfusion-associated reactions. Judicious selection of recipients and stringent follow-up after issue of blood units in wards especially ensuring optimal temperature, storage, transportation are essential. Hemovigilance provides scope for corrective and preventive action and gives referral guidelines to prevent future recurrence. It is believed that the practice of transfusing leukodepleted blood units in our hospital could enable to substantially retard the incidence of adverse events of transfusion as a general measure and febrile reactions in particular.

\section{Conclusion}

The present study is evident with a novelty in revealing the fact that the overall incidence of transfusion-associated reactions in the study domain is higher at very modest level when compared to more advanced centers in India. However, there were no significant variations observed with respect to cumulative incidence of ATRs based on the type of blood component transfused except for marginal association between packed cell transfusion and febrile reactions. There was neither delayed reaction nor mortality due to adverse reaction encountered in the study. The study analyzed the patterns of ATR with emphasis on management followed in treating adverse events as a unique aspect. The observations reiterate the use leukodepleted units in case of whole blood transfusion and packed cells to retard the incidence rate of adverse reactions. Hemovigilance assists toward inclination of quality assurance in blood bank and 
extends as a platform for fact finding rather than fault finding in analysis of ATRs.

\section{Conflict of Interest}

None declared.

\section{Acknowledgment}

The authors are grateful to technical staffs of blood bank, blood bank medical officer, staff nurses of clinical wards, and laboratory technicians.

\section{References}

1 IPC-NIB Guidance Document for Reporting Serious Adverse Reactions in Blood Transfusion Service. National Institute of Biological, Ministry of Health and Family Welfare, Government of India; 2012: Paper T1-31

2 Faber JC. Worldwide overview of existing haemovigilance systems. Transfus Apheresis Sci 2004;31(2):99-110

3 Faber JC. Haemovigilance around the world. Vox Sang 2002;83(suppl 1):71-76

4 Sahu S, Hemlata, Verma A. Adverse events related to blood transfusion. Indian J Anaesth 2014;58(5):543-551

5 Callum JL, Pinkerton PH, Transfusion reactions. In: Callum JL, Pinkerton PH, eds. Bloody Easy: Blood Transfusions, Blood Alternatives and Transfusion Reactions, A Guide to Transfusion Medicine. Toronto, Ontario: Sunny brook and Women's College Health Sciences Centre; 2005 34-65

6 Sharma DK, Datta S, Gupta A. Study of acute transfusion reactions in a teaching hospital of Sikkim: a hemovigilance initiative. Indian J Pharmacol 2015;47(4):370-374

7 Kumar P, Thapliyal R, Coshic P, Chatterjee K. Retrospective evaluation of adverse transfusion reactions following blood product transfusion from a tertiary care hospital: a preliminary step towards hemovigilance. Asian J Transfus Sci 2013;7(2):109-115
8 Payandeh M, Zare ME, Kansestani AN, et al. Descriptions of acute transfusion reactions in the teaching hospitals of Kermanshah University of Medical Sciences, Iran. Int J Hematol Oncol Stem Cell Res 2013;7(2):11-16

9 Venkatachalapathy TS. A prospective audit of blood transfusion reactions in tertiary care hospital for the use of blood and blood components. J Blood Disord Transfus 2012;3:118

10 Haslina MNN, Fakhri MA, Saw TH, Salamah AS. An audit on acute transfusion reaction in North Eastern Malaysia. Sch J Med 2012;2(5):60-62

11 Bhattacharya P, Marwaha N, Dhawan HK, Roy P, Sharma RR. Transfusion-related adverse events at the tertiary care center in North India: an institutional hemovigilance effort. Asian J Transfus Sci 2011;5(2):164-170

12 Kumar R, Gupta M, Gupta V, Kaur A, Gupta S. Acute transfusion reactions (ATRs) in intensive care unit (ICU): a retrospective study. J Clin Diagn Res 2014;8(2):127-129

13 Khalid S, Usman M, Khurshid M. Acute transfusion reactions encountered in patients at a tertiary care center. J Pak Med Assoc 2010;60(10):832-836

14 Mafirakureva N, Khoza S, Mvere DA. Chitiyo ME, Postma MJ, Van Hulst M. Incidence and pattern of 12 years of reported transfusion adverse events in Zimbabwe: a retrospective analysis. Blood Transfus 2014;12(3):362-367

15 Lingegowda JB, Jeyakumar JD, Muddegowda PH, Pitchai R, Gopal N, Sinha P. An audit of requests for fresh frozen plasma in a Tertiary Care Center in South India. J Lab Physicians 2016;8(1):41-44

16 Peña JR, Dzik WS. Utilization management in the blood transfusion service. Clin Chim Acta 2014;427:178-182

17 Agarwal N, Subramanian A, Pandey RM, Albert V, Karjee S, Arya V. An audit of fresh frozen plasma usage in a Tertiary Trauma Care Centre in North India. Indian J Hematol Blood Transfus 2014;30:328-332

18 Hendrickson JE, Hillyer CD. Noninfectious serious hazards of transfusion. Anesth Analg 2009;108(3):759-769

19 Adams RL, Bird R. Quality use of blood products. Aust Prescr 2014;37:132-136 